\title{
Healing Dysfunctional Identity: Bridging Mind-Body Intervention to Brain Systems
}

\author{
S. Shaun Ho ${ }^{1,2}$, Yoshio Nakamura ${ }^{3}$ \\ ${ }^{1}$ Department of Psychiatry, Stony Brook University, Stony Brook, NY, USA \\ ${ }^{2}$ Department of Psychiatry, University of Michigan, Ann Arbor, MI, USA \\ ${ }^{3}$ Department of Anesthesiology, Pain Research Center, University of Utah School of Medicine, Salt Lake City, UT, USA \\ Email: hosh@umich.edu,yoshi.nakamura@utah.edu
}

How to cite this paper: Ho, S.S. and $\mathrm{Na}$ kamura, Y. (2017) Healing Dysfunctional Identity: Bridging Mind-Body Intervention to Brain Systems. Journal of Behavioral and Brain Science, 7, 137-164. https://doi.org/10.4236/jbbs.2017.73013

Received: January 3, 2017

Accepted: March 24, 2017

Published: March 27, 2017

Copyright (c) 2017 by authors and Scientific Research Publishing Inc. This work is licensed under the Creative Commons Attribution International License (CC BY 4.0).

http://creativecommons.org/licenses/by/4.0/ cC (i) Open Access

\begin{abstract}
In this paper, we aim to use an innovative model to integrate applied work on a fast-acting mind-body intervention, Mind-Body Bridging (MBB), with theoretical work based on psychology and neuroscience. In an affect-object generative inference and regulation (AGIR) model, we propose that functional dynamics between two systems, the affect-object thought generation system and the cognitive control system, can guide an individual to achieve homeostasis within self and harmonious relationships with others. We used Neurosynth (www.neurosynth.org), an automated meta-analysis database, to identify potential brain substrates underlying the key components in the AGIR model. Based on the findings, some brain regions are implicated as the key cortical substrates in this model, corroborating our central hypothesis that a hallmark of mind-body wellbeing can be characterized as a low-frequency anti-correlantion between 1) the cognitive control system including the dorsal anterior/middle cingulate cortex, and 2) the affect-object thought generation system including the ventromedial prefrontal cortex and posterior cingulate cortex. MBB provides an efficient strategy for responding to and dissolving a fundamental problem that impairs mind-body wellbeing, i.e., unrealistic identity-grasping consisting of self-centered embodied expectations of self and others. We demonstrated how theoretical and applied work could be integrated by drawing evidence from the neuroscience literature to support the AGIR model, and then we applied the AGIR model to elucidate how MBB might work.
\end{abstract}

\section{Keywords}

Active Inference, Affects, Brain, Cortical Networks, Embodied Beliefs, Emotions, Emotion Regulation, Identity, Intervention, Mind-Body Wellbeing, Mind-Body Bridging, Neuroimaging, Psychopathology, Self 


\section{Introduction}

Emerging biological science suggests that life on Earth as a whole is maintained in a collective ecological homeostasis to which all organisms and environments contribute, and no organism can self-perpetuate and survive as a closed system, depending on nothing but its own genome [1]. In this symbiotic view of life, every organism is ever-changing and always dependant on other organisms [2]. Unless these conditions change in an evolving history of our cosmos, perhaps the most fundamental fallacy a human organism can make is identity-grasping, believing that there is a single independent identity, referred to as one's self, which is not changing and not dependent on anything else.

Why is this identity-grasping fallacy a problem for our current human conditions? For the whole ecological system, it is a problem because the ecological homeostasis as a whole will be undermined by erroneous efforts striving for individual homeostasis with a rigid identity, when the very singular identity is not grounded in any tangible, though ever-changing, biological and ecological processes. For a human being who holds a rigid expectation that his or her identity should always maintain its homeostasis in the exactly same way will sooner or later find it unrealistic when his or her internal and external milieus inevitably change. If the person does not relinquish this identity-grasping fallacy, he or she will be constantly anxious when being reminded of the likelihood of losing the identity in various forms and degrees. Eventually, the person becomes habitually obsessed with maintaining a certain identity that is threatened by the impermanence of life.

In the grips of such an obsession, individuals with identity-grasping see mental images of what they want or fear with reference to and in service of the grasped identities. According to the emerging literature of how consciousness arises, mental phenomena or images become part of one's subjective experience (a.k.a. consciousness), resulting from the "yoking" of top-down expectation and bottom-up data in the brain [3] [4]. The "yoking" not only occurs in a single unit of cortical area, called the cortical column, but also in long-range cortical-cortical connections between cortical networks [5] [6]. The yoking refers to a good enough match between 1) an expectation of what incoming data "should be", based on the previous consciousness, and 2) what data come to be perceived, when being matched against expectations. Importantly, the expectation of an imminent perception involves action planning, which is equivalent to an active inference of what would happen, given what has happened previously. In a generative model, these expectations inferred from prior probability are represented from a higher level hierarchy to predict the cause of the sensory information currently presented at the next lower level. Thus, mental phenomena in consciousness occur in a stream of frames of perception and expectation based on prior probability, yoked moment-by-moment. This kind of framework has been referred to as predictive coding or active inference models in many different domains: sensorimotor [7] [8], interoception [9], action selection [10], perceiving intention of others [11], and perception and action related to self and other [12]. In short, according to an active inference framework, consciousness arises depending on the yoking of per- 
ception and generative inference based on prior probability (action planning or expectation).

In light of this framework, we believe that neuroscience has the potential to offer an insight into the problem of the identity-grasping fallacy and its resolution, of which causal links can be described in terms of the following deductive logical reasoning: 1) From the identity-grasping fallacy some unrealistic expectations (a.k.a. action plans) ensue, 2) from unrealistic expectations realistic perceptions are erroneously missed while unrealistic perceptions are superstitiously reified, 3) from these mistakes in perceptions some erroneous actions are planned and taken, and 4) from mistaken actions some undesirable outcomes ensue. From experiencing undesirable outcomes, one arrives at a pivot point, either holding on to the momentum of ongoing unrealistic expectations and actions, or pausing and learning to correct mistakes and update expectations until they become realistically valid. Ultimately, only when an identity-grasping person fully recognizes that this identity-grasping is a fallacy too absurd to believe, undesirable outcomes stemming from the fallacy can cease to exist. This deductive reasoning based on an active inference framework is consistent with the Buddhist selflessness-oriented teachings [13] [14].

Meanwhile, it is important to find a practical entry point to intervene in everyday life to gradually eliminate unrealistic expectations and identity-grasping fallacy. Because cortical-cortical interactions in the brain subserve mental phenomena, which encompass not only unrealistic expectations but also conscious mind-body skills that can be intervened, we believe it is possible to use a cortical-cortical interaction model to account for the problem of identity-grasping and its interventions. In this current paper, we aim to provide a cortical-cortical interaction model to integrate with the applied work of healing dysfunctional identity-grasping. The paper consists of five parts: First, we will introduce the applied work of MindBody Bridging (MBB), which explicitly tackles the fallacy of identity-grasping. Second, we will bridge identity-grasping to the literature related to psychology of self-representations, self-defining memory, and embodied beliefs of interoception. Third, we will outline how embodied homeostasis-oriented active inference can generate affect-objects in an active inference system, and how it is normally regulated by a separate cognitive control system in a novel Affect-object Generative Inference and Regulation (AGIR) model. Supporting evidence will be provided from the neuroscience literature, including systematic meta-analytic reviews of imaging data available at Neurosynth.org. Fourth, we will integrate the AGIR model and the applied work of MBB. Lastly, we will discuss how our work is potentially applicable to a broader scope of mind-body wellbeing.

\section{Introduction of Mind-Body Bridging}

MBB is a short-term mind-body intervention informed by Western psychoanalysis and Eastern Zen-like contemplative practices. MBB employs innovative techniques to harness or curtail a common dysfunctional process presumably pervasive in psychological struggles [15] [16]. This dysfunctional process perpetuates 
maladaptive thoughts, feelings and behaviors and, in $\mathrm{MBB}$, is heuristically "personified" as an "Identity System" that can be activated when an individual encounters triggering situations or undesirable outcomes. MBB has been investigated as a short-term intervention for managing disturbed sleep and other co-occurring symptoms [17], as well as for dealing with various issues associated with substance misuse/abuse conditions, using $\mathrm{MBB}$ as a complementary intervention added to standard care [18]. Clinical trials have demonstrated that $\mathrm{MBB}$, compared to a mindfulness meditation condition, can be relatively more fast-acting and equally long-lasting in promoting mind-body wellbeing, after only three weekly sessions [19]. MBB currently is being investigated in two clinical trials with Veterans suffering from 1) Gulf War Illness and 2) mild Traumatic Brain Injury, presenting with disturbed sleep and other co-occurring symptoms.

The first objective of MBB is to develop skills to promote awareness of senses, feelings, and thoughts. These awareness practices serve two primary functions: first, to help the individual calm the mind and relax the body by stabilizing attention to focus and pausing spontaneous thoughts; second, to increase sensitivity or clarity in perceiving signs of a dysregulated state (referred to as an "active Identity System" state in MBB, denoted as IS+), such as subtle body tensions and racing thoughts, as well as signs of the absence of a dysregulated state (referred to as a "rested Identity System" state in MBB, denoted as IS-) such as mental clarity, calmness, and perceived cessation of body tension. To accomplish this goal, MBB incorporates an array of brief mindfulness exercises, including focused attention on a single object such as breathing, background sounds, or tactile sensations of fingers, as well as thought labeling, e.g., "I had a thought that [the content of the thought]." By doing these exercises, an individual can develop better attention control and more vivid awareness of sensations, feelings, and thoughts as mental phenomena, and thus can become more sensitive to the experiential differences between IS+ and IS- states.

The second objective of MBB is to develop an understanding of the "Identity System" in terms of its constituents and functions as experienced by an individual. Towards this objective, MBB employs several "mind-body mapping" exercises, which employ a technique of writing down thoughts in free associations related to a specific topic, with an explicit rating of associated body tensions for each identified thought; these verbal/non-verbal representations can promote sensitivity to signs of IS+ as experienced in an individual. One constituent of the "Identity System" is Requirements-expectations about how one and the world "should be" at a particular moment (e.g., "I should be respected by others")-that may be violated in a particular situation. MBB pedagogy asserts that the violation of Requirements can trigger a cascade of thought-based reactions called Depressor and Fixer that can form looping storylines by reciprocal reinforcement. Specifically, a Depressor reaction is characterized as a self-oriented critical thought that signifies a dissatisfaction towards one's self (e.g., "I am not respected because I'm not good enough"), and Fixer denotes a subsequent thought that magnifies the 
importance of a particular thought object projected to be what one needs to obtain or attain in order to satisfy the Depressor (e.g., "I need to make more money to be respected"). Depressor and Fixer thoughts can continue to occur spontaneously, regardless of whether one's achievement or relationship with others can support what these thoughts suggest. Consequently, Depressor and Fixer thoughts can reinforce each other in a habitual loop that does not stop running even after being invalidated by checking with reality. After an individual can recognize Requirement, Depressor, and Fixer, the MBB instructor introduces a process of "Defusing Requirements". In this process, an individual first develops awareness of "Requirements," then moving on to employ a broadened awareness to hold multiple elements involved in a triggering situation, including body sensations, feelings, and thoughts of freshly identified "Requirements," without any mental actions other then noticing (i.e., without trying to suppress any mental events or act out any thoughts). This "Defusing Requirement" strategy serves as an alternative response to a triggering situation by the combination of 1) conscious monitoring of mental events from their arising to dissolving, and 2) intentional inhibition of thoughts and urge-like responses.

Thus, for the purpose of this paper, we find clinical MBB pedagogy compatible with a potential brain-based active inference model, including unrealistic expectations (Requirements), symptoms of dysfunctional self-other perception and action (Depressors and Fixers), and situations involving undesirable outcomes (situations that violating Requirements). To advance towards a science of identitygrasping, in the next section we will introduce psychological and neuroscience processes potentially involved in identity-grasping.

\section{Towards a Science of Identity-Grasping}

\subsection{Bridging Identity-Grasping to Psychology of Self}

Identity-grasping as a problem has been at the core of modern psychotherapy theories. Clinical and developmental psychologists recognize that whether an individual develops a healthy sense of self depends on the person's developmental history since early childhood. Since birth, an infant develops what is called "self-object" to represent the caregiver and subsequently a representation of self. The development of objects of self and other (e.g., caregiver as "self-object") involves a "cathexis" process, which is to charge representations of self and others with affects. The child learns to control the caregiver "self-object" in a manner that is similar to that of learning to control his or her own mind and body, which is different from that of an adult seeking to seize control over another person [20]. In adulthood, a healthy person develops a cohesive self that can connect to others as a person independent of selfs needs rather than self-objects, and hence is enabled to relate to others for the sake of others [21], taking interests in others' success and welfare (i.e., empathy) [22] [23], which may decrease self-oriented identity-grasping.

However, a person who fails to develop a cohesive self may be prone to be overly obsessed with a vulnerable self (which is often manifested as the need to be reassured by others) and/or a failed self-object (which is often manifested as blaming 
a significant other who fails to provide what is expected), and thus both self and other can be represented as an object grasped as a fixed identity. When a discomfort arises, an identity-grasping individual can swing between two extreme ends of a narcissistic spectrum, with grandiosity and excitement on one end of the spectrum and shame and depression on the other end [20]. When a person perceives a threat that may injure the "grace" of his or her vulnerable self (i.e., the person may be affected by an uncomfortable "narcissist injury"), he or she will struggle to gain re-affirmation from others; this process is postulated to underlie most personality-related problems in an individual [24] and couples [25]. Consequently, identity-grasping can be a cause of excessive concerns for one's self-image as perceived by others, which can be related to unhealthy perfectionism [26] and self-criticism [27].

\subsection{Bridging Identity-Grasping to Self-Defining Autobiographical Memory}

From the perspective of cognitive psychology, the acquisition and retrieval of autobiographical memory have been postulated to play a key role in the generation of the phenomenal experience of "I" and the concept of identity [28]. It has been postulated that a frame of reference formed by the generation of narrative thoughts may enable a sense of presence, taken as one's self [29] and with reference to such presence. One can repeatedly generate a specific type of autobiographical memory, called self-defining memory, with the following qualities: affect-loaded, vividness, repeatedly rehearsed, strongly associated with similar memories, and, most relevantly here, motivationally connected with an enduring concern or unresolved conflict. This is considered to be a running script integrated in an individual's self-concepts [29]. Perhaps, identity-grasping may reflect a form of overrehearsed self-defining memories with reference to the sense of presence.

\subsection{Bridging Identity-Grasping to Embodied Beliefs in Interoception}

The self based on the felt sense of presence has been considered an embodied reference based on multiple sources of information, i.e., central, peripheral, autonomic nervous systems and molecular substrates of hormones and cytokines that can be referred to as internal milieu [9]. According to the Embodied Predictive Interoceptive Coding model [9], how an individual perceives the internal milieu is constrained by an embodied inference based on prior probability to predict how the individual would feel in a similar context. The inference is motivational as it is oriented to maintaining homeostasis. Such top-down embodied expectations are generated in the ventral part of the frontal lobe, and can be propagated to the middle or posterior insular cortex which receives incoming bottom-up data from the internal milieu to generate a felt sense (i.e., the subjective experience of "I feel ..."). The cortical areas that yoke top-down expectations and bottom-up data can measure any discrepancy between them, called prediction error, to be propagated to 1) feedback to the expectation generators to up- 
date top-down embodied expectations for the future, 2) trigger motor actions to change the relationship between organism and environment to adjust bottom-up data, and 3) adaptively use cognitive control to minimize consequences of prediction error.

It has been speculated that all emotions can be generated by the same principle, where simple core affects of pleasure or displeasure can be combined with other complex representations generated from conceptualization to give rise to emotions experienced in various flavors [30].

\section{Affect-Object Generative Inference and Regulation (AGIR) Model}

We postulate, in the AGIR model, that there should be an active inference model for the processes of grasping an identity, which is closely related to embodied beliefs of interoceptions and emotions.

\subsection{Components in the AGIR Model}

The AGIR model, as postulated here, operates in a domain of motivational affect-object processing, which is developed in early childhood for a young child to represent caregiver (as self-object) and subsequently self as mental objects in order to maintain homeostasis. The AGIR model consists of two systems, 1) an affect-object thought generation system oriented to maintaining homeostasis based on embodied expectations and object representations, and 2) a cognitive control system to resolve potential conflicts that may be or have been caused by unrealistic expectations.

The affect-object thought generation system is postulated to consist of affective potential generators, a proximal object sketchpad to represent self, a distal object sketchpad to represent others, and a thought generator to produce affect-object thoughts by combining affective potentials with represented objects. Like the active inference model for interoception [9], this affect-object thought generation system is an active inference system oriented to maintaining homeostasis. In this system, there are generators of homeostasis-oriented embodied beliefs, called affective potentials. These embodied beliefs represent how an individual should relate to self and/or others according to the beliefs on current homeostatic needs, which are primarily appetitive (anticipatory or consummatory feelings related to satisfaction or content) or aversive (anticipatory or consummatory feelings of harms or annoyance). As embodied beliefs, affective potentials can be generated and stored in the generator even before any object is represented in an object sketchpad. When representations of self and other are projected to object sketchpads as bottom-up data, affective potentials can be propagated to the object sketchpads also, as top-down expectations, to generate affect-objects that are subjectively experienced. The thought generator plays a critical role in generating and perpetuating affect-objects in mental phenomena, often with story-like narratives.

The cognitive control system is postulated to monitor all modalities of infor- 
mation and detect potential conflicts. With regard to thoughts generating affect-objects, if there are conflicting signals or predicting errors, the cognitive control system should pause the ongoing thoughts, observe broadly across multiple sources, and update affective potentials as top-down expectations accordingly.

A diagram of the AGIR model is depicted in Figure 1. For reasons explained later, each component is hypothesized to be preferentially related to a specific region of the brain: the precuneus/posterior cingulate cortex (PCC) for the thought generator; the subgenual anterior cingulate cortex (sgACC) and ventromedial prefrontal cortex (vmPFC) for the affective potential generator; the anteriomedial prefrontal cortex (amPFC) for the proximal object sketchpad; and the dorsomedial prefrontal cortex (dmPFC) for the distal object sketchpad in the affect-object system; and the dorsal ACC (dACC) and anteriodorsal middle cingulate cortex (adMCC) for the reality conflict monitor in the cognitive control system.

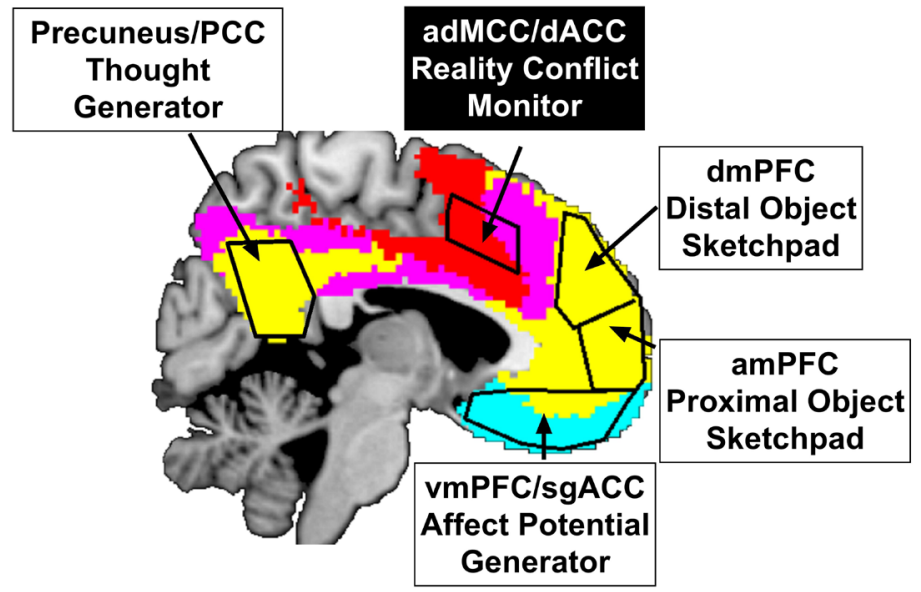

Figure 1. Midline cortical structures that are hypothesized as functional components in the affect-object generative inference \& regulation (AGIR) model. There are two systems in the model. First,a homeostasis-oriented affect-object inference generation system encompasses four components: a thought generator in precuneus/posterior cinculate cortex (PCC), an affect potential generator in subgenual anterior cingulate cortex (sgACC) for negative affect and ventromedial prefrontal cortex (vmPFC) for positive affect, a proximal object sketchpad in anterior medial prefrontal cortex (amPFC), and a distal object sketchpad in dorsomedial prefrontal cortex (dmPFC). When "vehicles" of thoughts are running in this system, thoughts of affect-objects can be represented as mental phenomena (e.g., self = proximal objects charged with affective potentials; other = distal objects charged with affective potentials). Second, a cognitive control system encompasses a component of reality conflict monitor in anterior dorsal middle cingulate cortex (adMCC)/dorsal anterior cingulate cortex (dACC) and other cortical components not depicted in the figure. The main function of reality conflict monitor is to pause an ongoing affect-object generating thought and observe all modalities of information involved in a potential conflict in reality, allowing an unrealistic expectation generated in the homeostasis-oriented system to be paused and updated. In terms of cortical networks that are self-organized in intrinsic resting-state functional connectivity in [31], the first system primarily involves Default Mode Network (yellow) and Cortical Limbic Network (cyan), and the second system primarily involves Ventral Attention Network (red) and Frontal-Parietal Network (violet).The colors of the components' text boxes are reversed between the two systems, indicating an anti-correlative relationship between them. The demarcation of the brain areas are approximate. 
Note that the demarcation of the regions is approximate and does not exclude non-midline structures' roles in these components. In addition to these components, Figure 1 also depicts four cortical networks identified based on intrinsic functional connectivity during resting state MRI scanning in 1000 healthy human brains [31]. Among these networks, the cortical limbic network (CLN) (indicated by a light blue shade) and the default-mode network (DMN) (indicated by a yellow shade) are postulated to subserve the functions of the affect-object system, i.e., generating perceptions of affect-object and perpetuating narrations involving interactions among multiple affect-objects in movie-like mental phenomena. Meanwhile, the frontal-parietal network (FPN) (indicated by a violet shade) and the ventral attention network (VAN) (indicated by a red shade) are postulated to subserve the functions of the cognitive control system, i.e., detecting conflicts between the affect-object system and other sources of information in reality, pausing the thoughts in the affect-object system, and observing and/or updating the original expectations imposed on affect-objects to reduce the prediction error and conflict with the reality.

It is postulated in the AGIR model that imminent incoherence between reality and ongoing affect-object thoughts will activate the reality conflict monitor, dACC/adMCC [32], which is part of the VAN identified in a previous study [31]. The VAN's function is typically to meta-cognitively monitor all relevant information by attributing them to respective sources when incoherence is significant. And the dACC/adMCC region has been specifically identified as a region in which the gray matter structural property is directly related to reality monitoring performance in a memory task to differentiate actually presented from imagined stimuli [33]. This dACC/adMCC region is of great clinical significance because structural and functional deficits of this region, along with the bilateral anterior insular cortex, are commonly found in multiple major psychiatric disorders including major depression, anxiety, obsessive-compulsive (OCD), substance use, bipolar, and schizophrenia disorders [34].

Besides reality conflict monitoring, as another part of the cognitive control system, the FPN's temporary connection with another network will be necessary for the information processed in that network to be generated as conscious mental phenomena [5]. For example, when individuals become subjectively aware of their spontaneous thoughts generated in the DMN, which may include affect-object thoughts, they will have co-activation of the DMN and FPN [35]. Likewise, the FPN's connectivity with the DMN will be strengthened when the participants are performing affect-object related tasks, either by retrieving autobiographical episodic memories from the past or imagining a future scene, but not when they are performing visual-spatial tasks unrelated to the function of the DMN [36].

\subsection{The AGIR Model's Central Hypothesis on Identity-Grasping}

According to the AGIR model, our central working hypothesis involves a provisional attempt to characterize an identity-grasping state that may harm mindbody wellbeing. That is, since affective potentials are embodied beliefs that may 
not necessarily reflect the actual homeostatic needs of self and others, if an individual grasps an identity rigidly, affective potentials will be generated as oriented to the homeostasis of the rigid identity, incongruent with the actual needs, and thus will lead the individual to develop and maintain unrealistic expectations. Meanwhile, upon combining biased affective potentials and representations of self and others, all affect-objects will be interpreted with reference to and in service of the grasped identity. Thus, the AGIR model postulates that an identity-grasping state is perpetuated by self-centered affect-objects thoughts, which are self-reinforcing, as these thoughts can only be confirmed by original embodied beliefs. The identity-grasping state seems to reflect multiple repeated failures of the cognitive control system in regulating the affect-object system, including both a failure in pausing the affect-object thoughts and a failure in updating affective potentials as embodied beliefs, since they are reinforced by currently running affect-object thoughts.

\subsection{Elucidating Brain Substrates of the AGIR Model}

To efficiently and qualitatively identify brain substrates potentially underlying the AGIR model, we utilized Neurosynth [37], which provides automated keyword-based meta-analysis for anyone accessing the database online (see http://www.neurosynth.org/analyses/terms/). By entering a term that exists in the Neurosynth database, one can see meta-analytically, in a sample of multiple neuroimaging studies, which brain areas are preferentially related to the term (keyword) in a reverse inference. That is, one can see whether it is statistically more probable to find a brain region reported in a number of neuroimaging papers that mentioned the keyword in the abstracts, as compared to papers included in the database that did not mention the keyword.

Although there can be no direct causality to indicate that a particular region is the basis of a construct that the keyword signifies, at least Neurosynth's reports can suggest that the absence of a particular brain region in a keyword-based reverse inference will mean the lack of specificity in associating the construct and the brain region in question. For example, when using "emotion" as a keyword, the amygdala, but not the PCC, is implicated in the reverse inference on Neurosynth. Conversely, when using "thinking" as a keyword, the PCC, but not the amygdala, is implicated. These examples combined illustrate the logic of "double dissociation", i.e., designating two different brain regions, the amygdala and PCC, to two different functions, emotion and thinking, respectively.

First, we need to identify multiple terms that produce substantial results in Neurosynth as the keywords for each of the AGIR model's components. We present this aspect of our work in Table 1, which tabulates the keywords selected, the number of studies using the keywords, the component(s) in the AGIR model relevant to the keywords, and descriptive rationales to map the keywords to components. The results in the Neurosynth meta-analysis based on the selected keywords are presented in Figure 2. Interested readers can replicate our results by entering http://www.neurosynth.org/analyses/terms/ [entered keyword] as a URL 
Table 1. Summary of mapping components of the AGIR model to keywords used in Neurosynth.

\begin{tabular}{|c|c|c|c|}
\hline Keyword & $\begin{array}{l}\text { Number of studies } \\
\text { with the keyword }\end{array}$ & $\begin{array}{l}\text { Relevant - } \\
\text { Component }(s)^{*}\end{array}$ & Rationale \\
\hline "thinking" & 110 & Component 1 & Thinking is the generation of thoughts. \\
\hline "autobiographical" & 108 & Component 1, 2 & $\begin{array}{l}\text { Thinking of autobiographical information should involve thought } \\
\text { generator and representations of self. }\end{array}$ \\
\hline "self-referential" & 127 & Component 1,2 & $\begin{array}{l}\text { Self-referential thoughts should involve thought generator and } \\
\text { representations of self as proximal objects. }\end{array}$ \\
\hline "retrieval" & 730 & Component 1,2 & $\begin{array}{l}\text { Retrieval is technically related to thinking of long-term memory and } \\
\text { holding it with immediate presence as proximal objects. }\end{array}$ \\
\hline "social cognition" & 166 & Component 1,3 & $\begin{array}{l}\text { Social cognition is typically related to perceiving and thinking of someone } \\
\text { else as distal objects. }\end{array}$ \\
\hline "mentalizing" & 124 & Component 1,3 & $\begin{array}{l}\text { Mentalizing refers to imagining another person's cognitive and emotional } \\
\text { states, thus involving thinking of distal objects. }\end{array}$ \\
\hline "beliefs" & 58 & Component 1,3 & $\begin{array}{l}\text { Beliefs is technically related to holding a belief that is not factual or } \\
\text { imagining someone else's beliefs, thus involving thinking of distal objects. }\end{array}$ \\
\hline “emotion" & 790 & Component 4 & Emotion is closely related to affective potentials. \\
\hline "reward" & 671 & Component 4 & $\begin{array}{l}\text { Reward is affective in terms of anticipatory or consummatory feelings of } \\
\text { satisfying homeostatic needs. }\end{array}$ \\
\hline “fear" & 298 & Component 4 & $\begin{array}{l}\text { Fear is affective in terms of anticipatory or consummatory feelings of } \\
\text { avoiding harms. }\end{array}$ \\
\hline "conflict" & 273 & Component 5 & $\begin{array}{l}\text { Conflict is technically related to conflicts between two alternative motor } \\
\text { responses. }\end{array}$ \\
\hline "monitoring" & 376 & Component 5 & $\begin{array}{l}\text { Monitoring is technically related to monitoring conflicts and ongoing - } \\
\text { information. }\end{array}$ \\
\hline
\end{tabular}

${ }^{*}$ Components 1 = Thought Generator, $2=$ Proximal Object Sketchpad, $3=$ Distal Object Sketchpad, $4=$ Affective Potential Generator, $5=$ Reality Conflict Monitor.

in an Internet browser.

To better demonstrate any functional dissociations between brain regions, we tabulated the keyword-based findings in a qualitative meta-summary, presented in Table 2. The shaded cells in Table 2 represent a region of interest (ROI) that is fully implicated in response to a set of keywords under a specific component of the AGIR model, i.e., the ROI is a common region underlying each and every keyword in a particular set. As it becomes clear in Table 2, all ROIs postulated in the AGIR model, except the PCC/precuneus designated as the Thought Generator, are doubly dissociated with one another, suggesting that these ROIs may be likely to subserve distinct functions as postulated in the AGIR model. While the PCC/precuneus is the best candidate as a brain substrate for the Thought Generator, it is also involved in proximal and distal object sketchpads, suggesting that the thought generator may generate objects in those sketchpads as part of mental phenomena. Nevertheless, the PCC/precuneus is not implicated in any of the keywords related to the Affective Potential Generator or Reality Conflict Monitor. Taken together, object-related thoughts and affective potentials are two functionally dissociable domains in the affect-object thought generation system and the reality conflict monitor is a key in the cognitive control system. 
A

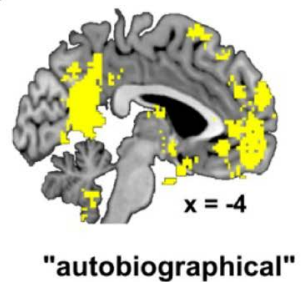

D

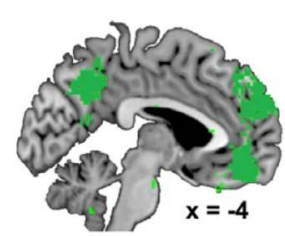

"mentalizing"

G

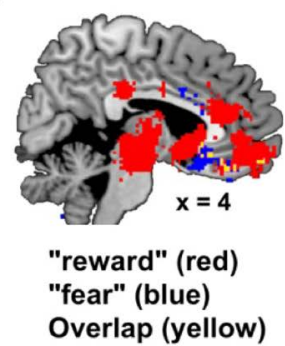

B

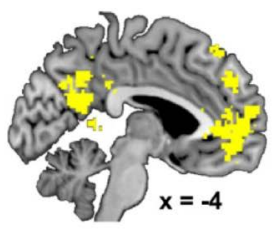

"self-referential"

E

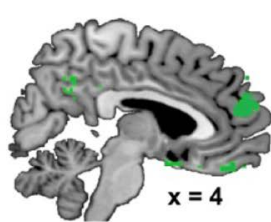

"social cognition"

H

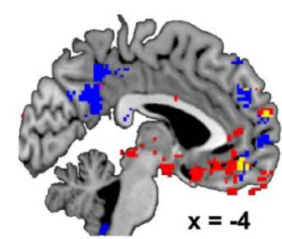

"emotion" (red)

"thinking" (blue)

Overlap (yellow)
C

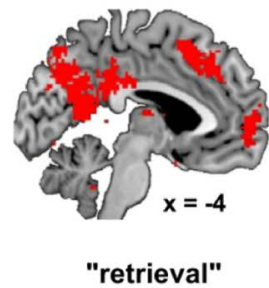

$\mathbf{F}$

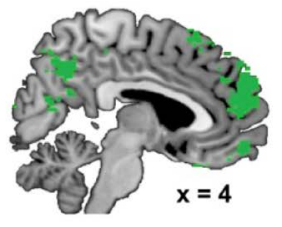

"beliefs"

I

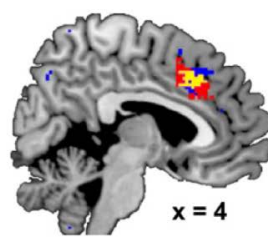

"conflict" (red)

"monitoring" (blue)

Overlap (yellow)

Figure 2. The results of keyword-based Neurosynth analyses are depicted in the midline areas, with keywords of "autobiographical" (A), "self-referential”(B), "retrieval" (C), "mentalizing"(D), "social cognition"(E), "beliefs"(F), "reward" in red, "fear" in blue, and overlap in yellowclusters $(G)$, "emotion" in red, "thinking" in blue, and overlap in yellowclusters $(\mathrm{H})$, and "conflict" in red, "monitoring" in blue, and overlap in yellowclusters (I). The implicated clusters were statistically significant in reverse reference related to the keyword, with whole brain correction at false-discovery rate at $\mathrm{p}<0.01$. The presence of a region of interest (ROI), as demarcated in Figure 1, suggests that this ROI is preferentially related to the keyword in a reverse inference, by being statistically more likely to be reported in articles that include the keyword in their abstracts than articles that do not. The absence of a ROI in the results suggests that this ROI is not specifically related to the keyword at all. The numbers at the bottom of brain image indicate their $\mathrm{x}$ coordinates in normalized MNI stereotaxic space.

\subsection{Functional Dynamics between Affect-Object and Cognitive Control Systems in the AGIR Model}

The central hypothesis mentioned above predicts a relatively stable anti-correlation between the two systems in the AGIR model. Using the traffic control in a city as a metaphor, there should be no single network in the brain that can continuously put vehicles of information running on the road without being stopped at intersections. According to the AGIR model, the affect-object thoughts are generated as "vehicles" running on intersecting roads, and these thoughts should be stopped and checked at the intersections to avoid or resolve conflict with vehicles generated from other modalities. Thus, we hypothesized that the activities of monitoring and potentially resolving conflict should aim to pause and therefore, 
Table 2. Qualitative meta-summary of the regions of interest and components of AGIR model in keyword-based meta-analysis in Neurosynth.

\begin{tabular}{|c|c|c|c|c|c|}
\hline & \multicolumn{5}{|c|}{ The AGIR Model's Regions of Interest (ROIs) } \\
\hline & 1 & 2 & 3 & 4 & 5 \\
\hline & PCC/Precuneus & amPFC & $\mathrm{dmPFC}$ & $\mathrm{sgACC} / \mathrm{vmPFC}$ & $\mathrm{dACC} / \mathrm{adMCC}$ \\
\hline 1. Thought Generator: & $(1,1)$ & & & & \\
\hline "thinking" & + & + & + & + & \\
\hline "autobiographical" & + & + & + & + & \\
\hline "self-referential" & + & + & + & + & \\
\hline "retrieval" & + & + & & & + \\
\hline "social cognition" & + & & + & & \\
\hline “mentalizing" & + & & + & + & \\
\hline "beliefs" & + & & + & + & \\
\hline 2. Proximal Object Sketchpad (Self): & $(1,2)$ & $(2,2)$ & & & \\
\hline "autobiographical" & + & + & + & + & \\
\hline "self-referential" & + & + & + & + & \\
\hline "retrieval" & + & + & & & + \\
\hline 3. Distal Object Sketchpad (Other): & $(1,3)$ & & $(3,3)$ & & \\
\hline "social cognition" & + & & + & & \\
\hline "mentalizing" & + & & + & + & \\
\hline "beliefs" & + & & + & + & \\
\hline 4. Affective Potential Generator: & & & & $(4,4)$ & \\
\hline “emotion” & & & + & + & \\
\hline "reward" & & & & + & \\
\hline "fear" & & & & + & \\
\hline 5. Reality Conflict Monitor: & & & & & $(5,5)$ \\
\hline “conflict” & & & & & + \\
\hline “monitoring" & & & & & + \\
\hline
\end{tabular}

Note: ' + ' indicates affirmation that a brain region is preferentially related to a specific keyword and a blank cell indicates a negation thereof based on keyword-based automated meta-analysis in Neurosynth. A shaded "meta-cell" indicates a Region of Interest (ROI) that is preferentially related to a component in AGIR, as it is conjunctively related to all the keywords that are associated with that particular component in this analysis. Focusing on the ROIs' shaded "meta-cells" noted with respective coordinates of (ROI number, component number), double dissociations can be observed in varying degrees: Meta-cells 2 - 5 are doubly dissociated with one another (the shaded cells are positioned only in the diagonal of a $4 \times 4$ box formed by ROIs 2-5 and components $2-5$ ); meta-cell 1 is doubly dissociated with meta-cells $4 \& 5$.

inhibit activities of generating homeostasis-oriented thoughts. In other words, these networks should have an anti-correlated relationship.

To test this hypothesis, we examined the functional connectivity with the dACC/adMCC seed, placed at MNI coordinates $(4,20,36)$, defined as the conjunction between the results of [34] and the Neurosynth analysis of "conflict" and "monitoring" (Figure 2(I)), by utilizing the resting state fMRI database in 1000 healthy young participants [31] available on Neurosynth. Interested readers can replicate our finding by entering http://www.neurosynth.org/locations/4_20_36_6/ as URL in an Internet brows- 
er. This analysis revealed that the dACC/adMCC seed was anti-correlated with the vmPFC, PCC, and several other DMN areas, while it is positively correlated with other nodes in VAN (Figure 3). These results support the postulation that healthy individuals normally alternate between a) actively generating homeostasis-oriented predictions relating to self and others (subserved by vmPFC and PCC) and b) pausing these predictions for reality check (subserved by dACC/ adMCC) and any necessary update (subserved by cognitive control networks).

Interestingly, dACC/adMCC was not anti-correlated with dmPFC or amPFC during the resting state, suggesting that, while reality monitoring can directly inhibit thought and affect generations, and vice versa, there is no reciprocal opponent relationship between reality monitoring and representations of self and others in the respective object sketchpads. This postulation is consistent with the experiential phenomena in the practice of open monitoring meditations, wherein a practitioner merely observes the qualia of mental phenomena as representations in any modalities, which is potentially demonstrated as temporary positive correlations between the VAN and DMN (perhaps only amPFC or dmPFC nodes but not vmPFC) during this type of meditation [38].

Since the anti-correlated relationship between the VAN and DMN was consistently found in the low-frequency range $(<0.1 \mathrm{~Hz})$ during states of resting as well as active tasks [39], perhaps the rate of such between-network alternation (oscillation) is around 10 seconds or longer per cycle. Interestingly, this anti-correlation

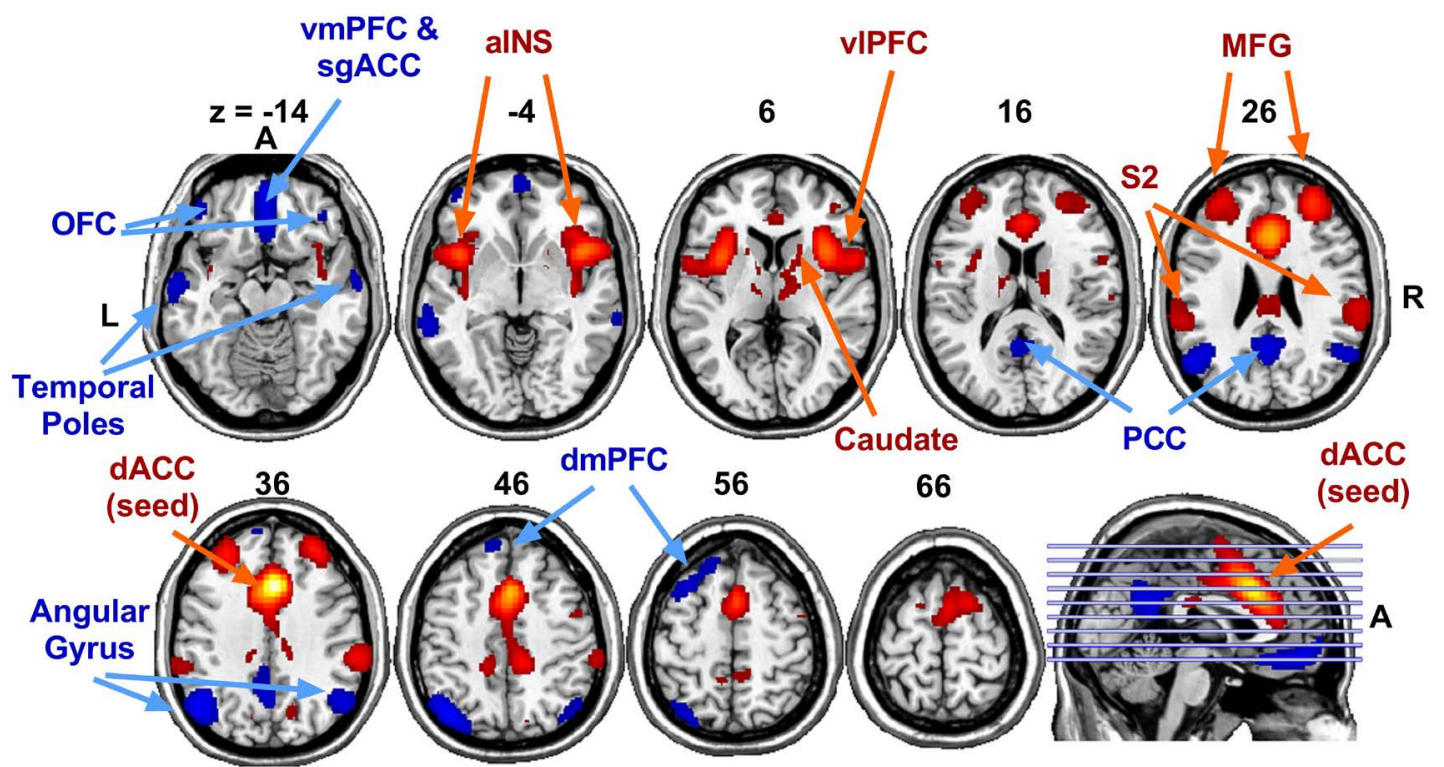

Figure 3. Horizontal 2D slices of brain images showing the results in resting-state functional connectivity analysis in 1000 healthy invidividuals, with the dACC seed located at MNI152 coordinates (4, 20, 36), as generated in an open-access datebase (http://www.neurosynth.org/locations/4_20_36_6/). The red and blue clusters are those with positive and negative correlations with the seed, respectively. aINS: anterior insular cortex; dACC: dorsal anterior cingulate cortex; dmPFC: dorsomedial prefrontal cortex; MFG: middle frontal gyrus; OFC: orbitofrontal cortex; sgACC: PCC: posterior cingulate cortex; subgenual anterior cingulate cortex; vlPFC: ventrolateral prefrontal cortex; vmPFC: ventromedial prefrontal cortex. The numbers on the top of slices indicate their $\mathrm{z}$ coordinates in normalized MNI stereotaxic space and alphabets indicate directions: A, anterior; L, left side; $R$, right side. 
oscillates at a rate similar to the rate of breathing that can induce respiratory sinus arrhythmia, a state of maximal coherence between breathing and heart rate variability that generates stress-reducing effects [40]. Not surprisingly, the brain regions participating in this oscillation, the $\mathrm{dACC} / \mathrm{adMCC}$, vmPFC, and PCC, were also cortical substrates for autonomic regulation [41]. Thus, the anti-correlation between the dACC/adMCC and vmPFC and PCC at $0.1 \mathrm{~Hz}$ is related to the multiple major hallmarks of mind-body wellbeing.

Conversely, the central AGIR hypothesis predicts that the lack of coordination between the reality monitor ( $\mathrm{ACC} / \mathrm{adMCC}$ ) and the active generators of affect-object thoughts (vmPFC and PCC) will be the hallmark of such dysfunctional unhealthy states. Indeed, the diminished anti-correlation between the VAN and DMN and excessive activities in the DMN have been reported consistently as another emerging signature of the lack of mind-body wellbeing [42] [43] [44] [45], in addition to the aforementioned data [34].

\subsection{Some Considerations on the Brain Substrates of the AGIR Model}

For interested readers, in this section we wanted to offer further in-depth discussions based on specific empirical studies, to better ascertain the functional roles of the ROIs as postulated in the AGIR model. With regard to the PCC, thinking is an active action that constructs propositions and stories in thoughts. When the PCC is dysregulated, excessive thoughts may fill up the mind. A study utilizing the first-person narratives of the meditating participants suggested that the better the participants can stop thinking, the better they can inhibit the activity in the PCC; conversely, PCC activity was related to the spontaneous thoughts when these participants were effortfully contriving to self regulate [46]. Indeed, meditations that enhance attentional focus and stability are found to inhibit PCC activity [47]. Thus, we postulate that the PCC/precuneus node is the best candidate for thought generator and, further, it is the thought generator (PCC/precuneus) that actively closes a circuit to charge social objects represented in $\mathrm{dmPFC}$ and amPFC with the affective potentials generated in vmPFC.

With regard to vmPFC/sgACC, according to the meta-summary of the Neurosynth analysis in Table 2, vmPFC/sgACC as a substrate for affective potential generator can be functionally dissociated with the thought generator PCC. Interestingly, it was documented in a study that when performing a reward learning task with a thought of playing a card game with a hypothetical "opponent", the participants' vmPFC can respond to the same reward signal positively when it is received by self but negatively when received by the hypothetical opponent, when there is no direct competition between the two players at all [48]. These results suggest that the affective potentials generated in vmPFC can be framed to be self-centered preceding the actual contact of objects, which is consistent with the notion that affective potentials are expectations or beliefs oriented to maintaining homeostasis, based on what an individual believes to be desirable in a specific context, not necessarily reflecting what the raw data mean (i.e., what reality is). Notably, when an animal's appetite for salt is experimentally induced, 
the animal will immediately seek a very salty solution when cued for the availability of the solution, even though the solution has been tasted and always refused by the animal in the past [49]. This intriguing observation is consistent with our model that the affective potential is produced based on an embodied inference to maintain homeostasis of the internal milieu, so when the homeostasis calls for unusually high amount of salt, very salty water will be represented as a desirable affect-object.

With regard to amPFC, a plausible reason to regard amPFC (also known as BA10p) as a brain substrate for proximal object sketchpad is due to its intimate links to interoception-processing areas such as the insular and anterior cingulate cortices, along with other multimodal higher-order cognitive processing [50]. Functionally, the activity in amPFC during the retrieval of negative autobiographical memories has been found to show graded activations according to the degrees of grasping of these memories [51], along with other key regions of interest in our working model. Thus, the literature supports our postulation that amPFC serves as a proximal object sketchpad to represent self with a vivid felt sense.

It is noteworthy that there are several studies supporting our distinction of amPFC and dmPFC as proximal and distal object sketchpads. When viewing aversive images, excessive amPFC and vmPFC activations were found in psychotic patients with symptoms of reality distortion, compared to healthy individuals, but excessive dmPFC activation was found in psychotic patients without reality distortion [52]. This is consistent with the model that $\mathrm{dmPFC}$ is related to the construction of distal object representations, which by itself does not have a gripping sense of being real, so the patients without symptoms of reality distortion can still recognize that it was not real. Conversely, the amPFC may represent a gripping sense of reality with affective potentials from the vmPFC in patients with symptoms of reality distortion. Furthermore, when manipulating conditions of retrieving autobiographical memory versus imagining a future social scene, both conditions activated the PCC and precuneus, but the memory retrieval condition activated the amPFC and the future imagination condition activated the dmPFC [36]. Taken together, these results are consistent with our functional designations of the amPFC representing proximal objects with reality-like quality and the dmPFC representing distal objects with imagination-like quality.

Moreover, the distal object (other) represented in the dmPFC may be viewed by the individual as "an object related to me" rather than "a person independent of me." For example, the dmPFC was more activated when individuals predicted what others might do on the basis of a belief that was not differentiated from the self's perspective (undifferentiated belief), as compared to what they might do on the basis of a belief that was differentiated, when the other's perspective would be opposite to the self's (differentiated belief); conversely, the dACC/adMCC, right prefrontal cortex, right temporal parietal junction, and right dorsal precuneus were more activated in differentiated belief than undifferentiated belief conditions [53]. This study provides supportive evidence that the dmPFC represents another person's attributes as a distal object in a self-centered perspective, 
while the $\mathrm{dACC} / \mathrm{adMCC}$ is needed when one represents others as different from one's self. Interestingly, seeing another person in a self-centered perceptive, i.e., self-object, is what developmental theory of psychopathology would consider as a symptom of under-developed self that lacks empathy [22] [23].

An additional Neurosynth analysis implicated both the sgACC and dmPFC with the term "negative affect", which is consistent with the sgACC's postulated function in negative affective potential, but not any other ROIs in the AGIR model, including the anterior part of the vmPFC for positive affective potential, the amPFC for proximal object sketchpad, the PCC for thought generation, and the dACC/adMCC for reality monitoring. As excessive dmPFC activity during a resting state has been found in the patients with major depression disorder [54], our designation of the $\mathrm{dmPFC}$ as a sketchpad for representing distal objects from a self-centered perspective is consistent with its roles in the rumination of interpersonal stories that are quite prevalent in depressed patients [55]. When affective potential generated from the vmPFC is connected to representations of others in the dmPFC, these others as social objects will appear in one's mental phenomena with affective flavors of liked or disliked person (e.g., social appraisal of others) [56]. Thus, these results suggest that most unpleasant feelings an individual may perceive may result from projecting negative affective potentials in the sgACC onto a distal object represented in the dmPFC, which manifests as displeasure directed against someone else. This may explain the apparent irritability in patients with mood disorders or other mind-body illnesses [57].

Lastly, with regard to the dACC/adMCC as a substrate for reality conflict monitor, the results of Neurosynth analysis are highly consistent with a study showing that individual differences in this brain area are directly related to the capacity to correctly monitor reality [33], and such individual differences are related to cognitive control of negative affects as well [58]. Furthermore, a meta-analysis of meditation studies suggested that the activity in the dACC/adMCC area is enhanced by meditations that improve meta-cognitive awareness of mental phenomena (i.e., open monitoring meditation) and attentional focus and stability (i.e., focused meditation) [47].

\section{Bridging Dys-Regulation of Affect-Object Thoughts to Applied Work}

\subsection{Dys-Regulation of the AGIR Model}

Drawing from the central hypothesis of the AGIR model, repeated failures in pausing affect-object thoughts when embodied beliefs result in conflicts can result in failures in updating the embodied beliefs generated as affective potentials. In a vicious cycle, un-updated embodied beliefs further fuel affect-object thoughts in order to regain the expected homeostasis, even though these embodied beliefs have led to the conflicts already. In this process, apparently there is a sensitization of negative affective potentials accumulated in the sgACC and vmPFC. For the sgACC, the negative affective potential refers to the presence of harm or absence of safety. For the vmPFC, the negative affective potential refers to 
the absence of anticipated reward or cessation of satisfaction. Sensitization refers to a phenomenon in which the same amount of stimulus can trigger increasingly stronger responses when repeated, e.g., a small error can trigger stronger irritations upon repetitions.

What may cause the failures in pausing affect-object thoughts after prediction errors or conflicts? And what may cause the sensitization of negative affective potentials after prediction errors or conflicts? According to the AGIR model, potentiated reactivity in the $\mathrm{PCC}$ and $\mathrm{sgACC} / \mathrm{vmPFC}$ as the generators of thoughts and negative affective potentials may directly inhibit the dACC/ adM$\mathrm{CC}$, due to the anti-correlation between the two networks. The inhibition of the dACC/adMCC as reality conflict monitor can in turn dis-inhibit the PCC and sgACC/vmPFC, thus making these generators more sensitized.

But what has to become aberrant before the anti-correlation between the VAN and DMN can become diminished chronically? To trace the cause of dys-regulation, we may rely on the early symptoms of dys-regulated affect-object thoughts. One of the most common early symptoms in psychopathology is the sensitization of urge-to-react, such as the development of sensitized reaction triggered by a specific stimulus (i.e., a previously neutral stimulus or cue becomes very potent in provoking unhealthy reactions, such as irritation or anger, and urges to react, seeking or avoiding certain objects) [59]. We may liken urge-to-react to allergic inflammations triggered by allergens. Thus, we postulate that urge-to-react after conflicts or prediction errors may potentiate the negative affective potentials, and then by sufficient repetition, the urge-to-react can trigger full blown allergy-like behavioral reactions, including dys-regulated affect-object thoughts.

One of the most influential theories related to various urge-like reactions is the Incentive Salience Sensitization Theory [60], developed in the science of drug addiction [61] and behavioral addictions such as gambling [62]. The incentive salience refers to an object acquiring emotional and/or motivational values (appetitive or aversive) and thus becoming a salient object. The cathexis process of connecting emotional energies to an object underlies the development of incentive salience, as originally theorized by Edward C. Tolman [63] and others [60] [64]. Likewise, cathexis is a central process involved in the generation of affect-object, when embodied beliefs as affective potentials are combined with object representations in the AGIR model.

The caudate, a sub-cortical region in the brain, may play a key role in urge-toreact and its effects on cortical networks. In a healthy state, prediction errors can activate the caudate [65], and then the caudate can activate urge-to-react [66]. When the urge becomes strong, it can increase response in the sgACC and vmPFC along with increasing craving for substances (e.g., nicotine) [67]. Compared to healthy individuals, the patients with OCD showed excessive coupling between the caudate and midline frontal areas such as the sgACC, vmPFC, and dmPFC [68]. Thus, it is likely that a dys-regulated prediction error can result in an urge-toreact with excessive caudate activation and functional coupling between the caudate and the sgACC, vmPFC, and dmPFC. This postulation is consistent with a neuro- 
imaging study showing that, when facing a fear-evoking situation, stronger coupling between the caudate and sgACC resulted in greater urge and unrealistic reactions [69].

Interestingly, the aforementioned neuroimaging study [69] also showed that stronger coupling between the caudate and right ventrolateral prefrontal cortex (vlPFC) resulted in less urge and no unrealistic reactions. The right vlPFC, located at the junction between the VAN and FPN, is known to mediate cognitive control of emotions [70], and it is part of the clusters that are positively correlated with the dACC/adMCC seeded resting-state functional connectivity (Figure 3). These results suggest that, in a healthy state, the caudate participated in the cognitive control system in the AGIR model, along with the reality monitoring $\mathrm{dACC} / \mathrm{adMCC}$ and emotion regulating right vlPFC.

\subsection{Brain-Based Model for Mind-Body Bridging}

Now we are finally ready to integrate the practical work in MBB with the brain-based model of affect-object regulation. Recall that MBB considers that a dysfunctional self will manifest as an activated Identity System with symptoms of urge-like body tension and/or racing thoughts, violated Requirements, and storylines involving ruminative self-criticizing Depressor thoughts and objectseeking Fixer thoughts. MBB instructors teach patients to use paper-and-pencil exercises to "map" out the tension-related thoughts in order to recognize Requirements, Depressors, and Fixers, which are heuristically labeled as Identity System elements. Through informal daily mindfulness/awareness practices, the patients sharpen their ability to recognize body tensions and racing thoughts as soon as they are triggered in a situation, a state referred to as activated Identity System. Then, in a technique called defusing Requirements, the patients learn to pause their reactions by broadening their awareness to all sensory inputs from the present moment, while concurrently observing inwardly any emerging Requirements and verbally labelling them as Requirement thoughts in order to attenuate self-immersion in thoughts.

In a healthy IS- state, an individual can possess healthy dynamics between the affect-object and cognitive control systems. In such a state, when conflicts suggest a potential Requirement is being violated, both an initial urge to react and the underlying Requirement can be recognized and defused. Overall, the healthy anti-correlation between the VAN and DMN is maintained, and both Requirement-driven thinking and affective potential propelling Depressor/Fixer are inhibited. This is illustrated in the lower loop in Figure 4.

Conversely, in an unhealthy IS+ state, an individual encounters a failure in pausing thoughts and updating predictions that have been in conflict with reality. The thoughts perpetuated in a dysfunctional state are loaded with affect-objects viewed in a self-centered perspective, and they can be identity-defining, similar to self defining memories that are affect-loaded, vivid, repeatedly rehearsed, strongly associated with similar memories or concepts, or motivationally connected with an enduring concern or unresolved conflict [29]. When the Identity 


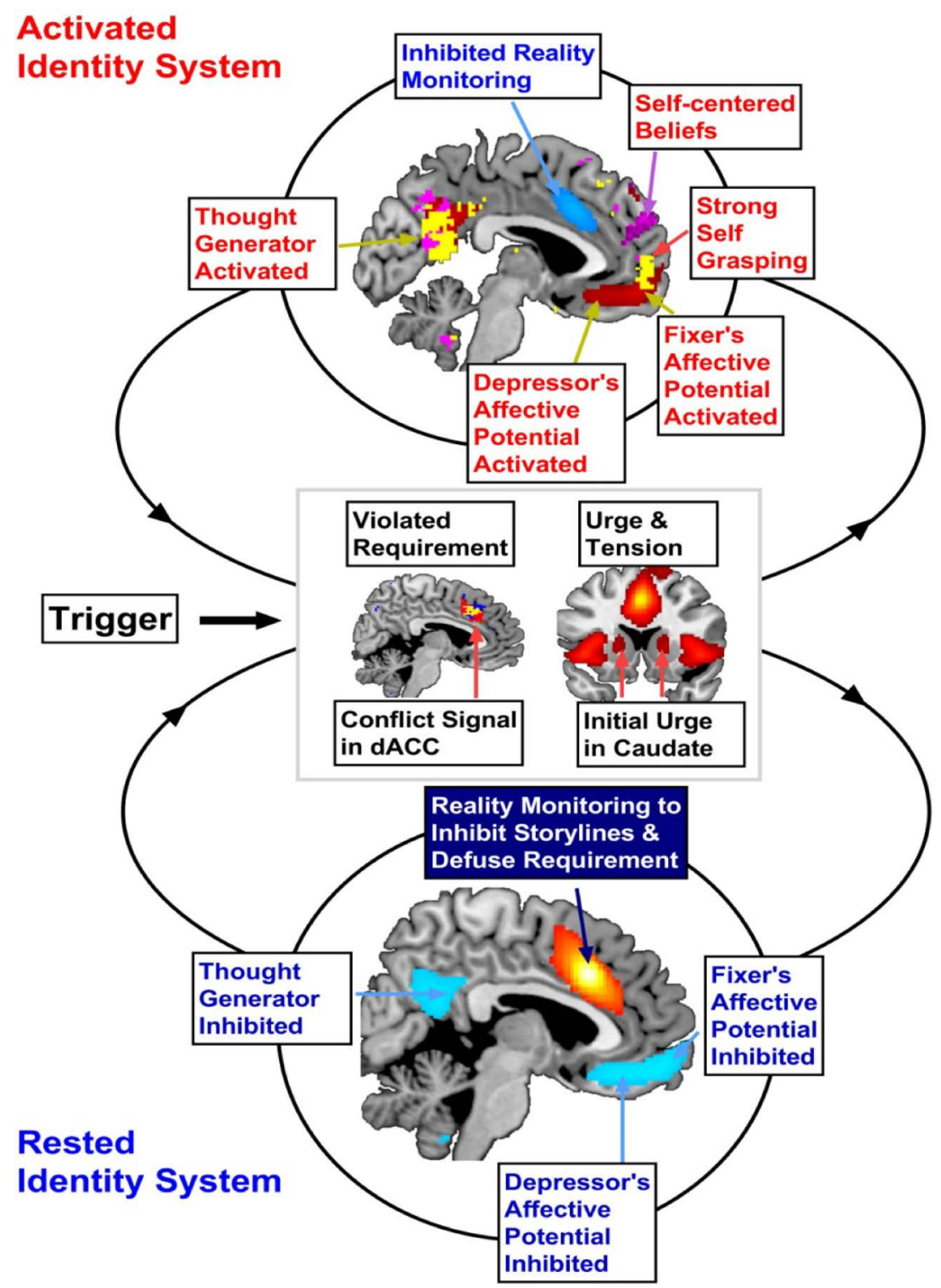

Figure 4. Elucidating Mind-Body Bridging (MBB) processes in light of AGIR model. When a situation involves a potential conflict between reality and an unrealistic expectation (Requirement), it may trigger early symptoms of mind-body dysregulation, e.g., urge and body tension. A state of mind-body dysregulation may ensue, if these early symptoms are not recognized, affective potentials for Depressor-Fixer and thoughts are not inhibited, and original expectations are not updated. Alternatively, if early symptoms are monitored and Requirement is defused, dysfunctional thoughts and affective potentials will be inhibited to facilitate adaptive mind-body wellbeing. In MBB, a dysregulated state is refered to as an activated Identity System (IS+), depicted in the upper half of the figure. Alternatively, the absence of a dysregulated state is referred to as a rested Identity System (IS-), depicted in the lower half of the figure.

System is activated by an apparent violation of a Requirement in a triggering situation, the $\mathrm{dACC} / \mathrm{adMCC}$ fails to adequately monitor the conflict, leading to uncontrolled automatic urge-like reactions in the caudate. The caudate is excessively engaged with the PCC/Precuneus (generating storylines), vmPFC (representing negative affective potentials, i.e., Depressor, and positive affective poten- 
tials for external objects, i.e., Fixer), amPFC (immersed in gripping sense of self defining memory), and dmPFC (excessive self-centered beliefs about the way self and others should be). Thus, thoughts are perpetuated as storylines instead of being paused by dACC/adMCC. This is illustrated in the upper loop in Figure 4.

The intervention of MBB aims to develop a healthy alternative response to prediction errors that have been triggering the Identity System. Foundational work with mindfulness/awareness practices has been found to up-regulate the $\mathrm{dACC} / \mathrm{adMCC}$ that can monitor prediction errors or response conflicts and down-regulate the PCC/Precuneus [47]. Thus, MBB informal awareness practice may strengthen the normal anti-correlation between the dACC/adMCC and storyline generating PCC/Precuneus and vmPFC (Figure 3), such that when prediction errors activate the $\mathrm{AACC} / \mathrm{adMCC}$ and caudate initially, the Identity System will be less likely to be activated.

Furthermore, as MBB practitioners learn to defuse Requirements through the practice of cognitively mapping Requirements, Depressors, and Fixers related to urge-like tensions, the initial activation of the caudate may not lead to excessive urge. We postulate that this Requirement defusing process involves the following. According to a neuroimaging study, when facing a fear-evoking situation, stronger coupling between the caudate and right ventrolateral prefrontal cortex (vlPFC) resulted in less urge and no unrealistic reactions, but stronger coupling between the caudate and sgACC resulted in greater urge and unrealistic reactions [69]. Meanwhile, the right vlPFC has been implicated in affect labeling that can reduce negative emotional responses in the amygdala [71], and the sgACC is related to fear and depression and postulated as the Depressor part of the vmPFC. Thus, MBB provides a leverage, similar to affective labeling, to name an urge-laden emotional process as an "activated Identity System" such that initial urge-like tension and racing thoughts related to a latent Requirement can be cognitively managed and, eventually, original unrealistic expectations may be updated and thus defuse the Requirement.

\section{Concluding Remarks}

In this paper, we attempted to integrate theoretical work in psychology and neuroscience with applied work with $\mathrm{MBB}$, a fast-acting mind-body intervention. The AGIR model suggests that a person can generate embodied beliefs (expectations) to infer what should happen in relation to self and others. The negative and positive embodied expectations generated in the sgACC and vmPFC, respectively, serve as affective potentials ready to emotionally charge any objects that may be projected onto object sketchpads.

Using Neurosynth, we found that the neuroimaging literature suggested that there are two affective homeostasis-oriented systems, a defensive system related to "fear" and a wanting system related to "reward", which are both part of the cortical limbic network and the adjacent part of the DMN (Figure 2(G)). These affective potentials remain pre-conceptual as mood, until they are connected with an explicit object by a mental action initiated in the PCC/precuneus, i.e., "thinking" 
of self and others (Figure 2(G)). The thinking action functionally connects affective potentials from the vmPFC with representations of objects in the amPFC (for proximal objects that are related to one's own identity) and the dmPFC (for distal objects that are not related to self identity, e.g., others) to produce affect-objects being enacted in self-centered thoughts.

Importantly, so long as affective potentials are referenced to self-centered homeostasis, the affect-objects of self and others are going to be viewed from a self-centered perspective with a goal of solely optimizing homeostasis for the sake of the self's internal milieu, regardless of symbiotic relationships with others. Thus, even when an individual in this state is thinking about another person, this person is probably represented as a distal affect-object charged with negative affect potentials, instead of other-person as the target of empathy. The lack of empathy is not easily recognized by a self-centered individual, because negative affective potentials can be pre-conceptual (i.e., generated before a thought is generated), and be propagated to forwardly generate a view of self as a proximal affect-object represented in the amPFC. In combination, proximal and distal affect-objects generated by embodied unrealistic expectations misinform an individual about his or her internal milieu and moods, resulting in unrealistically fabricated thoughts.

This process of unrealistic affect-object fabrication may explain why individuals with high levels of alexithymia (symptoms of mis-reading or under-recognizing their own interoceptive feelings) are at risk for mind-body illness and inflammation [72] [73], and impairments in social emotional functioning [74] [75]. People with high levels of alexithymia showed impairment in the anterior cingulate cortex and insular cortex in the brain [76], the key nodes in the VAN and the substrates of the cognitive control system in the AGIR model.

Can MBB mitigate alexithymia? A strategy that is consistent with MBB practice and the AGIR model is to facilitate access to a valid readout of the actual internal milieus to update erroneous embodied beliefs. Alexithymia may be a clinical condition in which future research could tackle a mind-body problem in light of the integration between theoretical and applied work presented here. Many neuropsychiatric conditions involves symptoms of alexithymia. For example, alexithymia was associated with apathy and companion's distress in patients with neurodegenerative diseases [77] and neurodegenerative diseases implicated FPN and DMN atrophy [78]. While MBB effects on FPN, VAN, and DMN cortical networks remain to be tested in the future, we hope our presentation of MBB as practice and the AGIR model as neuroscience-informed theory will be useful in stimulating readers to continue to search for resolutions (or dissolutions) for dysfunctional problems originating in the counter-productive embodiment of identity-grasping fallacy.

\section{Acknowledgements}

The authors acknowledge receiving financial support for efforts contributing to the current article from I-System, Inc. (to S.S.H.) and the Department of Defense 
(W81XWH-12-1-0385 to Y.N.). The authors confirm that, in full compliance with the bylaws of the authors' affiliated institutions, none of the funding providers influenced the results reported in the article or the write-up of the article.

\section{References}

[1] Gilbert, S.F., Bosch, T.C.G. and Ledon-Rettig, C. (2015) Eco-Evo-Devo: Developmental Symbiosis and Developmental Plasticity as Evolutionary Agents. Nature Reviews Genetics, 16, 611-622. https://doi.org/10.1038/nrg3982

[2] Gilbert, S.F., Sapp, J. and Tauber, A.I. (2012) A Symbiotic View of Life: We Have Never Been Individuals. The Quarterly Review of Biology, 87, 325-341.

https://doi.org/10.1086/668166

[3] Bachmann, T. and Hudetz, A.G. (2014) It Is Time to Combine the Two Main Traditions in the Research on the Neural Correlates of Consciousness: $\mathrm{C}=\mathrm{L} \times \mathrm{D}$. Frontiers in Psychology, 2014. 5. https://doi.org/10.3389/fpsyg.2014.00940

[4] He, B.J. and Raichle, M.E. (2009) The fMRI Signal, Slow Cortical Potential and Consciousness. Trends in Cognitive Sciences, 13, 302-309. https://doi.org/10.1016/j.tics.2009.04.004

[5] Cole, M.W., Reynolds, J.R., Power, J.D., Repovs, G., Anticevic, A. and Braver, T.S. (2013) Multi-Task Connectivity Reveals Flexible Hubs for Adaptive Task Control. Nature Neuroscience, 16, 1348-1355. https://doi.org/10.1038/nn.3470

[6] Mashour, G.A. (2013) Cognitive Unbinding: A Neuroscientific Paradigm of General Anesthesia and Related States of Unconsciousness. Neuroscience \& Biobehavioral Reviews, 37, 2751-2759. https://doi.org/10.1016/j.neubiorev.2013.09.009

[7] Friston, K. (2010) The Free-Energy Principle: A Unified Brain Theory? Nature Reviews Neuroscience, 11, 127-138. https://doi.org/10.1038/nrn2787

[8] Hommel, B., Müsseler, J., Aschersleben, G. and Prinz, W. (2001) The Theory of Event Coding (TEC): A Framework for Perception and Action Planning. Behavioral and Brain Sciences, 24, 849-878. https://doi.org/10.1017/S0140525X01000103

[9] Barrett, L.F. and Simmons, W.K. (2015) Interoceptive Predictions in the Brain. Nature Reviews Neuroscience, 16, 419-429. https://doi.org/10.1038/nrn3950

[10] Friston, K.J., et al. (2012) Dopamine, Affordance and Active Inference. PLOS Computational Biology, 8, e1002327. https://doi.org/10.1371/journal.pcbi.1002327

[11] Kilner, J.M., Friston, K.J. and Frith, C.D. (2007) Predictive Coding: An Account of the Mirror Neuron System. Cognitive Processing, 8, 159-166. https://doi.org/10.1007/s10339-007-0170-2

[12] Jordan, J.S. (2003) Emergence of Self and Other in Perception and Action: An Event-Control Approach. Consciousness and Cognition, 12, 633-646. https://doi.org/10.1016/S1053-8100(03)00075-8

[13] Tenzin Gyatso, T.T.D.L. (1997) The Four Noble Truths: Fundamentals of Buddhist Teachings. Thorsons, London.

[14] Tenzin Gyatso, T.T.D.L. (2009) The Middle Way Faith Grounded in Reason. Wisdom Publications, New York.

[15] Block, S. and Block, C.B. (2002) Bridging the I-System: Unifying Spirituality and Behavior. White Cloud Press, Ashland.

[16] Block, S. and Block, C.B. (2007) Come to Your Senses: Demystifying the Mind-Body Connection. Atria Books/Beyond Words, Hillsboro.

[17] Nakamura, Y., Lipschitz, D.L., Landward, R., Kuhn, R. and West, G. (2011) Two Sessions of Sleep-Focused Mind-Body Bridging Improve Self-Reported Symptoms of 
Sleep and PTSD in Veterans: A Pilot Randomized Controlled Trial. Journal of Psychosomatic Research, 70, 335-345. https://doi.org/10.1016/j.jpsychores.2010.09.007

[18] Nakamura, Y., Lipschitz, D.L., Kanarowski, E., McCormick, T., Sutherland, D. and Melow-Murchie, M. (2015) Investigating Impacts of Incorporating an Adjuvant Mind-Body Intervention Method into Treatment as Usual at a Community-Based Substance Abuse Treatment Facility. SAGE Open, 5, 1-18.

https://doi.org/10.1177/2158244015572489

[19] Nakamura, Y., Lipschitz, D.L., Kuhn, R., Kinney, A.Y. and Donaldson, G.W. (2013) Investigating Efficacy of Two Brief Mind-Body Intervention Programs for Managing Sleep Disturbance in Cancer Survivors: A Pilot Randomized Controlled Trial. Journal of Cancer Survivorship, 7, 165-182. https://doi.org/10.1007/s11764-012-0252-8

[20] Kohut, H. (2009) The Analysis of the Self: A Systematic Approach to the Psychoanalytic Treatment of Narcissistic Personality Disorders. University of Chicago Press, Chicago. https://doi.org/10.7208/chicago/9780226450148.001.0001

[21] Batson, C.D., Ahmad, N. and Lishner, D.A. (2009) Empathy and Altruism. In: Lopez, S. and Snyder, C., Eds., The Oxford Handbook of Positive Psychology, Oxford University Press, Oxford, New York, 417-426.

https://doi.org/10.1093/oxfordhb/9780195187243.013.0039

[22] Kohut, H. (1982) Introspection, Empathy, and the Semi-Circle of Mental-Health. International Journal of Psycho-Analysis, 63, 395-407.

[23] Summers, F. (2014) The Bonds of Empathy: Beyond the Self-Object Concept. International Journal of Psychoanalytic Self Psychology, 9, 222-236. https://doi.org/10.1080/15551024.2014.917464

[24] Kohut, H. (1972) Thoughts on Narcissism and Narcissistic Rage. Psychoanalytic Study of the Child, 27, 360-400.

[25] Carlson, J. and Sperry, L. (1998) The Disordered Couple. Brunner/Mazel, New York.

[26] Hill, A.P. and Curran, T. (2015) Multidimensional Perfectionism and Burnout: A Meta-Analysis. Personality and Social Psychology Review, 20, 269-288.

[27] Gilbert, P. and Procter, S. (2006) Compassionate Mind Training for People with High Shame and Self-Criticism: Overview and Pilot Study of a Group Therapy Approach. Clinical Psychology \& Psychotherapy, 13, 353-379. https://doi.org/10.1002/cpp.507

[28] Addis, D.R. and Tippett, L.J. (2010) The Contributions of Autobiographical Memory to the Content and Continuity of Identity. In: Sani, F., Ed., Self-Continuity: Individual and Collective Perspectives, Psychology Press, New York, 71-84.

[29] Conway, M.A., Singer, J.A. and Tagini, A. (2004) The Self and Autobiographical Memory: Correspondence and Coherence. Social Cognition, 22, 491-529. https://doi.org/10.1521/soco.22.5.491.50768

[30] Lindquist, K.A. and Barrett, L.F. (2012) A Functional Architecture of the Human Brain: Emerging Insights from the Science of Emotion. Trends in Cognitive Sciences, 16, 533-540. https://doi.org/10.1016/j.tics.2012.09.005

[31] Yeo, B.T., et al. (2011) The Organization of the Human Cerebral Cortex Estimated by Intrinsic Functional Connectivity. Journal of Neurophysiology, 106, 1125-1165. https://doi.org/10.1152/jn.00338.2011

[32] De la Vega, A., Chang, L.J., Banich, M.T., Wager, T.D. and Yarkoni, T. (2016) Large-Scale Meta-Analysis of Human Medial Frontal Cortex Reveals Tripartite Functional Organization. The Journal of Neuroscience, 36, 6553-6562. 
https://doi.org/10.1523/JNEUROSCI.4402-15.2016

[33] Buda, M., Fornito, A., Bergström, Z.M. and Simons, J.S. (2011) A Specific Brain Structural Basis for Individual Differences in Reality Monitoring. The Journal of Neuroscience, 31, 14308-14313. https://doi.org/10.1523/JNEUROSCI.3595-11.2011

[34] Goodkind, M., et al. (2015) Identification of a Common Neurobiological Substrate for Mental Illness. JAMA Psychiatry, 72, 305-315. https://doi.org/10.1001/jamapsychiatry.2014.2206

[35] Fox, K.C., et al. (2015) The Wandering Brain: Meta-Analysis of Functional Neuroimaging Studies of Mind-Wandering and Related Spontaneous Thought Processes. Neuroimage, 111, 611-621. https://doi.org/10.1016/j.neuroimage.2015.02.039

[36] Schacter, D.L., Addis, D.R., Hassabis, D., Martin, V.C., Spreng, R.N. and Szpunar, K.K. (2012) The Future of Memory: Remembering, Imagining, and the Brain. Neuron, 76, 677-694. https://doi.org/10.1016/j.neuron.2012.11.001

[37] Yarkoni, T., Poldrack, R.A., Nichols, T.E., Van Essen, D.C. and Wager, T.D. (2011) Large-Scale Automated Synthesis of Human Functional Neuroimaging Data. Nature Methods, 8, 665-670. https://doi.org/10.1038/nmeth.1635

[38] Josipovic, Z., Dinstein, I., Weber, J. and Heeger, D.J. (2012) Influence of Meditation on Anti-Correlated Networks in the Brain. Frontiers in Human Neuroscience, 5, 183. https://doi.org/10.3389/fnhum.2011.00183

[39] Kong, F., Hu, S., Wang, X., Song, Y. and Liu, J. (2015) Neural Correlates of the Happy Life: The Amplitude of Spontaneous Low Frequency Fluctuations Predicts Subjective Well-Being. NeuroImage, 107, 136-145. https://doi.org/10.1016/j.neuroimage.2014.11.033

[40] McCraty, R. and Zayas, M.A. (2014) Cardiac Coherence, Self-Regulation, Autonomic Stability, and Psychosocial Well-Being. Frontiers in Psychology, 5, 1090.

[41] Beissner, F., Meissner, K., Bär, K.-J. and Napadow, V. (2013) The Autonomic Brain: An Activation Likelihood Estimation Meta-Analysis for Central Processing of Autonomic Function. The Journal of Neuroscience, 33, 10503-10511. https://doi.org/10.1523/JNEUROSCI.1103-13.2013

[42] Baliki, M.N., Geha, P.Y., Apkarian, A.V. and Chialvo, D.R. (2008) Beyond Feeling: Chronic Pain Hurts the Brain, Disrupting the Default-Mode Network Dynamics. The Journal of Neuroscience, 28, 1398-1403. https://doi.org/10.1523/JNEUROSCI.4123-07.2008

[43] Hamilton, J.P., Furman, D.J., Chang, C., Thomason, M.E., Dennis, E. and Gotlib, I.H. (2011) Default-Mode and Task-Positive Network Activity in Major Depressive Disorder: Implications for Adaptive and Maladaptive Rumination. Biological Psychiatry, 70, 327-333. https://doi.org/10.1016/j.biopsych.2011.02.003

[44] MacNamara, A., DiGangi, J. and Phan, K.L. (2016) Aberrant Spontaneous and Task-Dependent Functional Connections in the Anxious Brain. Biological Psychiatry. Cognitive Neuroscience and Neuroimaging, 1, 278-287. https://doi.org/10.1016/j.bpsc.2015.12.004

[45] De Havas, J.A., et al. (2012) Sleep Deprivation Reduces Default Mode Network Connectivity and Anti-Correlation during Rest and Task Performance. NeuroImage, 59, 1745-1751. https://doi.org/10.1016/j.neuroimage.2011.08.026

[46] Garrison, K.A., Santoyo, J.F., Davis, J.H., Thornhill IV, T.A., Kerr, C.E. and Brewer, J.A. (2013) Effortless Awareness: Using Real Time Neurofeedback to Investigate Correlates of Posterior Cingulate Cortex Activity in Meditators' Self-Report. Frontiers in Human Neuroscience, 7, 440. https://doi.org/10.3389/fnhum.2013.00440

[47] Fox, K.C., et al. (2016) Functional Neuroanatomy of Meditation: A Review and Me- 
ta-Analysis of 78 Functional Neuroimaging Investigations. Neuroscience \& Biobehavioral Reviews, 65, 208-228. https://doi.org/10.1016/j.neubiorev.2016.03.021

[48] Ho, S.S., et al. (2012) Neurocircuits Underlying Cognition-Emotion Interaction in a Social Decision Making Context. Neuroimage, 63, 843-857.

https://doi.org/10.1016/j.neuroimage.2012.07.017

[49] Robinson, M.J. and Berridge, K.C. (2013) Instant Transformation of Learned Repulsion into Motivational "Wanting”. Current Biology, 23, 282-289. https://doi.org/10.1016/j.cub.2013.01.016

[50] Petrides, M. and Pandya, D.N. (2007) Efferent Association Pathways from the Rostral Prefrontal Cortex in the Macaque Monkey. The Journal of Neuroscience, 27, 11573-11586. https://doi.org/10.1523/JNEUROSCI.2419-07.2007

[51] Kross, E., Davidson, M., Weber, J. and Ochsner, K. (2009) Coping with Emotions Past: The Neural Bases of Regulating Affect Associated with Negative Autobiographical Memories. Biological Psychiatry, 65, 361-366. https://doi.org/10.1016/j.biopsych.2008.10.019

[52] Taylor, S.F., Welsh, R.C., Chen, A.C., Velander, A.J. and Liberzon, I. (2007) Medial Frontal Hyperactivity in Reality Distortion. Biological Psychiatry, 61, 1171-1178. https://doi.org/10.1016/j.biopsych.2006.11.029

[53] Sommer, M., et al. (2007) Neural Correlates of True and False Belief Reasoning. NeuroImage, 35, 1378-1384. https://doi.org/10.1016/j.neuroimage.2007.01.042

[54] Sheline, Y.I., Price, J.L., Yan, Z. and Mintun, M.A. (2010) Resting-State Functional MRI in Depression Unmasks Increased Connectivity between Networks via the Dorsal Nexus. Proceedings of the National Academy of Sciences, 107, 11020-11025. https://doi.org/10.1073/pnas.1000446107

[55] Lemogne, C., Delaveau, P., Freton, M., Guionnet, S. and Fossati, P. (2012) Medial Prefrontal Cortex and the Self in Major Depression. Journal of Affective Disorders, 136, e1-e11. https://doi.org/10.1016/j.jad.2010.11.034

[56] Liberzon, I., Ma, S.T., Okada, G., Ho, S.S., Swain, J.E. and Evans, G.W. (2015) Childhood Poverty and Recruitment of Adult Emotion Regulatory Neurocircuitry. Social Cognitive and Affective Neuroscience, 10, 1596-1606. https://doi.org/10.1093/scan/nsv045

[57] Fava, G.A., Cosci, F. and Sonino, N. (2017) Current Psychosomatic Practice. Psychotherapy and Psychosomatics, 86, 13-30. https://doi.org/10.1159/000448856

[58] Shackman, A.J., Salomons, T.V., Slagter, H.A., Fox, A.S., Winter, J.J. and Davidson, R.J. (2011) The Integration of Negative Affect, Pain and Cognitive Control in the Cingulate Cortex. Nature Reviews Neuroscience, 12, 154-167. https://doi.org/10.1038/nrn2994

[59] Pelchat, M.L. (2002) Of Human Bondage: Food Craving, Obsession, Compulsion, and Addiction. Physiology \& Behavior, 76, 347-352. https://doi.org/10.1016/S0031-9384(02)00757-6

[60] Robinson, T.E. and Berridge, K.C. (1993) The Neural Basis of Drug Craving: An Incentive-Sensitization Theory of Addiction. Brain Research Reviews, 18, 247-291. https://doi.org/10.1016/0165-0173(93)90013-P

[61] Berridge, K.C., Robinson, T.E. and Aldridge, J.W. (2009) Dissecting Components of Reward: "Liking", "Wanting", and Learning. Current Opinion in Pharmacology, 9, 65-73. https://doi.org/10.1016/j.coph.2008.12.014

[62] Robinson, M.J., et al. (2014) Initial Uncertainty in Pavlovian Reward Prediction Persistently Elevates Incentive Salience and Extends Sign-Tracking to Normally Unattractive Cues. Behavioural Brain Research, 266, 119-130. 
https://doi.org/10.1016/j.bbr.2014.03.004

[63] Tolman, E.C. (1945) A Stimulus-Expectancy Need-Cathexis Psychology. Science, 101, 160-166. https://doi.org/10.1126/science.101.2616.160

[64] Dickinson, A. and Balleine, B. (1994) Motivational Control of Goal-Directed Action. Animal Learning and Behavior, 22, 1-18. https://doi.org/10.3758/BF03199951

[65] O’Doherty, J., Dayan, P., Schultz, J., Deichmann, R., Friston, K. and Dolan, R.J. (2004) Dissociable Roles of Ventral and Dorsal Striatum in Instrumental Conditioning. Science, 304, 452-454. https://doi.org/10.1126/science.1094285

[66] Jackson, S.R., Parkinson, A., Kim, S.Y., Schüermann, M. and Eickhoff, S.B. (2011) On the Functional Anatomy of the Urge-for-Action. Cognitive Neuroscience, 2, 227-243. https://doi.org/10.1080/17588928.2011.604717

[67] Wilson, S.J. and Sayette, M.A. (2015) Neuroimaging Craving: Urge Intensity Matters. Addiction, 110, 195-203. https://doi.org/10.1111/add.12676

[68] Harrison, B.J., et al. (2009) Altered Corticostriatal Functional Connectivity in Obsessive-Compulsive Disorder. Archives of General Psychiatry, 66, 1189-1200. https://doi.org/10.1001/archgenpsychiatry.2009.152

[69] Gillan, C.M., et al. (2015) Functional Neuroimaging of Avoidance Habits in Obsessive-Compulsive Disorder. The American Journal of Psychiatry, 172, 284-293. https://doi.org/10.1176/appi.ajp.2014.14040525

[70] Wager, T.D., Davidson, M.L., Hughes, B.L., Lindquist, M.A. and Ochsner, K.N. (2008) Prefrontal-Subcortical Pathways Mediating Successful Emotion Regulation. Neuron, 59, 1037-1050. https://doi.org/10.1016/j.neuron.2008.09.006

[71] Lieberman, M.D., et al. (2007) Putting Feelings into Words: Affect Labeling Disrupts Amygdala Activity in Response to Affective Stimuli. Psychological Science, 18, 421-428. https://doi.org/10.1111/j.1467-9280.2007.01916.x

[72] Honkalampi, K., et al. (2011) Alexithymia and Tissue Inflammation. Psychotherapy and Psychosomatics, 80, 359-364. https://doi.org/10.1159/000327583

[73] Mattila, A.K., et al. (2008) Alexithymia and Somatization in General Population. Psychosomatic Medicine, 70, 716-722. https://doi.org/10.1097/PSY.0b013e31816ffc39

[74] Moriguchi, Y., et al. (2007) Empathy and Judging Other's Pain: An fMRI Study of Alexithymia. Cerebral Cortex, 17, 2223-2234. https://doi.org/10.1093/cercor/bhl130

[75] Van der Velde, J., et al. (2013) Neural Correlates of Alexithymia: A Meta-Analysis of Emotion Processing Studies. Neuroscience \& Biobehavioral Reviews, 37, 1774 1785. https://doi.org/10.1016/j.neubiorev.2013.07.008

[76] Kano, M. and Fukudo, S. (2013) The Alexithymic Brain: The Neural Pathways Linking Alexithymia to Physical Disorders. BioPsychoSocial Medicine, 7, 1. https://doi.org/10.1186/1751-0759-7-1

[77] Sturm, V.E. and Levenson, R.W. (2011) Alexithymia in Neurodegenerative Disease. Neurocase, 17, 242-250. https://doi.org/10.1080/13554794.2010.532503

[78] Li, H.-J., et al. (2015) Toward Systems Neuroscience in Mild Cognitive Impairment and Alzheimer's Disease: A Meta-Analysis of 75 fMRI Studies. Human Brain Mapping, 36, 1217-1232. https://doi.org/10.1002/hbm.22689 


\section{Abbreviations}

adMCC: Anteriodorsol middle cingulate cortex

AGIR Model: Affect-object Generative Inference and Regulation Model amPFC: Anteriomedial prefrontal cortex

BA: Brodmann Area

CLN: Cortical limbic network

dACC: Dorsal anterior cingulate cortex

DMN: Default mode network

dmPFC: Dorsomedial prefrontal cortex

IS+: Active Identity System

IS-: Rested Identity System

MBB: Mind Body Bridging

FPN: Frontoparietal network

PCC: Posterior cingulate cortex

ROI: Region of interest

sgACC: Subgenual anterior cingulate cortex

VAN: Ventral attention network

vlPFC: Ventrolateral prefrontal cortex

vmPFC: Ventromedial prefrontal cortex

\section{Scientific Research Publishing}

Submit or recommend next manuscript to SCIRP and we will provide best service for you:

Accepting pre-submission inquiries through Email, Facebook, LinkedIn, Twitter, etc.

A wide selection of journals (inclusive of 9 subjects, more than 200 journals)

Providing 24-hour high-quality service

User-friendly online submission system

Fair and swift peer-review system

Efficient typesetting and proofreading procedure

Display of the result of downloads and visits, as well as the number of cited articles

Maximum dissemination of your research work

Submit your manuscript at: http://papersubmission.scirp.org/

Or contact jbbs@scirp.org 